\title{
Degradación térmica de polimetíl metacrilato en múltiples extrusiones
}

CHEMICAL ENGINEERING

\section{Thermal degradation of polymethyl methacrylate in multiple extrusions}

\author{
Andrés F. Rojas*§, Laura M. Aranzazu*, Guillermo H. Gaviria*, Javier I. Carrero* \\ * Departamento de Ingeniería Química, Facultad de Ingeniería y Arquitectura, Universidad Nacional \\ de Colombia Sede Manizales, Manizales, Colombia \\ §anfrojasgo@unal.edu.co,lmaranzazur@unal.edu.co,ghgavirial@unal.edu.co,licarrerom@unal.edu.co,
}

Recibido: 13 de Marzo de 2013 - Aceptado: 06 de junio de 2014

\begin{abstract}
Resumen
Se presentan los resultados del estudio cinético de la degradación térmica del Polimetíl Metacrilato - PMMA, tanto virgen como procesado por extrusión hasta cinco ciclos de reprocesamiento, en una extrusora monohusillo. Después de cada reprocesamiento se tomó una muestra para análisis. A estas muestras se les determinó la temperatura de transición vítrea (por DSC), índice de fluidez y la temperatura máxima de pérdida de peso (por TGA). Los resultados del análisis termogravimétrico se utilizaron para determinar el triplete cinético por medio de métodos isoconversionales, no isoconversionales, y dinámicos. Se encontró que las curvas TG, DTG y de conversión, se desplazan a la derecha al aumentar la velocidad de calentamiento. También se encontró que los métodos no isoconversionales diferenciales presentaron valores de energía de activación mayores a los métodos integrales. La energía de activación aumentó con el reprocesamiento y la probabilidad o fracción de colisión aumenta con la velocidad de calentamiento.
\end{abstract}

Palabras claves: Degradación térmica de polímeros, múltiples extrusiones, triplete cinético.

\begin{abstract}
This work presents a kinetic study of thermal degradation of polymethyl metacrylate (PMMA). This material was subject of five extrusion cycles in a single barrel screw extruder, and results for raw and reprocessed material are presented. Glass transition temperature (by DSC), melt flow index, and temperature of maximum weight loss rate (by TGA) were determined in samples taken after each reprocessing. Results from thermogravimetric analysis were used to get the kinetic triplet by isoconversional, non-isoconversional, and dynamic methods as it is reported in literature. It was found that heating shifts to the right TG, DTG, and conversion curves. Activation energy values found with non-isoconversional differential methods tend to be higher than those found with integral methods. Also the activation energy increased with reprocessing, while the probability or collision factor increased with the heating rate.
\end{abstract}

Key words: Multiple extrusions, thermal degradation of polymers, triplet kinetic. 


\section{Introducción}

La degradación de un polímero es el proceso irreversible mediante el cual se produce una disminución significativa en sus propiedades mecánicas, volviéndolo quebradizo y dando como consecuencia una reducción de la vida útil del material (Jitendra, 2005). Esto es causado por factores externos como: luz, temperatura, esfuerzos mecánicos, entre otros, los cuales experimentan los materiales poliméricos durante su uso (Brandrup, 1996), o su procesamiento (Pérez, 2010). Esta degradación se presenta por fotodegradación, biodegradación, degradación ultrasónica, degradación de alta energía causado por energía de radiación y/o termodegradación (Jitendra, 2005). La termodegradación o degradación térmica de los polímeros es causada por las condiciones de calentamiento y temperatura, la cual se puede determinar por Análisis Termogravimétrico del material, al comparar los termogramas entre el polímero virgen y el polímero reprocesado. Como resultado del Análisis Termogravimétrico se tiene la curva de pérdida de peso versus temperatura, o curva TG, y su derivada se conoce como diagrama termogravimétrico diferencial o curva DTG (Grellmann \& Seidler, 2007). La curva DTG proporciona información de si un polímero se degrada más fácilmente (menor temperatura pico, $\mathrm{T}_{\mathrm{m}}$ ) que otro, al comparar la temperatura a la máxima velocidad de pérdida de peso, Tm (Carrasco et al, 2010).

La termodegradación causada por el reprocesamiento ocasiona cambios en la temperatura de transición vítrea $(\mathrm{Tg})$, peso molecular e índice de fluidez del polímero (IF) (Wilichowski, 2004). Por lo cual, se ha establecido que cuando un polímero se degrada por rompimiento de la cadena principal, su peso molecular y viscosidad se reducen y IF aumenta (Wilichowski, 2004). Se ha encontrado que la Tg en el policarbonato disminuye con el número de ciclos de reprocesamiento (Pérez et al., 2010). En cuanto a la cristalinidad, en el polietileno no fue afectada por el reprocesamiento (Abad et al., 2004), mientras que en polipropileno se incrementa (Martins \& DePaoli, 2002). Este mismo comportamiento se observó con materiales compuestos como poliuretano/bagazo (Mothé et al., 2002). Respecto al IF, se ha encontrado que en el polietileno de alta densidad grado extrusión aumentaconel número deciclos dereprocesamiento en una extrusora (Wilichowski, 2004), mientras que al reprocesar en inyección el IF disminuye (Mendes et al., 2011), esto indica que el polímero se degradó por el mecanismo de reticulado, el cual considera que las moléculas se entrecruzan provocando dificultad en su movilidad. Para el policarbonato se reporta que el IF aumenta con el número de ciclos de procesamiento en inyección y en extrusión, siendo mayor en inyección (Pérez et al., 2010). Resultados similares se reportan al reprocesar polipropileno en extrusión (Martins $\&$ De Paoli, 2002). También se ha encontrado que durante la degradación térmica de PMMA a $250^{\circ} \mathrm{C}$, se tiene como productos principales de la degradación moléculas de monómero, agua, $\mathrm{CO}, \mathrm{CO}_{2}$, compuestos alifáticos y aldehídos (Holland \& Hay, 2001). Esto sugiere que agua, $\mathrm{CO}$ y $\mathrm{CO}_{2}$, serán los productos principales de la termodegradación de PMMA, al experimentar varias extrusiones consecutivos, debido no solamente a la degradación del polímero, sino también al monómero, compuestos alifáticos y aldehídos.

La termodegradación de polímeros se puede estudiar a través de su cinética, obteniendo el triplete cinético, para lo cual actualmente se cuenta en la literatura con aproximadamente 50 métodos de análisis de datos obtenidos por TGA. Estos métodos son clasificados en forma general, en métodos isoconversionales y no isoconversionales. Los métodos isoconversionales, a diferencia de los no isoconversionales, fijan la conversión entre 0 y 1 , no necesitan asumir el modelo o la cinética de la reacción, los parámetros cinéticos se obtienen en función de la conversión o la temperatura, y no son función de la velocidad de calentamiento. Los modelos no isoconversionales obtienen un valor de el triplete cinético para todo el rango de conversión (Dahiya et al., 2008).

El objetivo de este trabajo es estudiar la cinética de degradación térmica (triplete cinético) del PMMA tanto virgen como reprocesado a través de 5 ciclos consecutivos de procesamiento 
en extrusión, empleando datos del análisis termogravimétrico y utilizando los métodos de Flynn-Wall-Ozawa, Friedman, Gyulai- Greenhow, Kissinger-Akahira-Sunose y Kissinger, como modelos isoconversionales, y los métodos Diferencial e Integral de Arrhenius, Briodo, Chang, Coats-Redfern, Flynn-Wall, Horowitz-Metzger, MacCallum-Tanner, Madhusudanan y el método Dinámico, como modelos no isoconversionales.

\section{Metodología}

Características del polímero. Para este estudio se utilizó polimetíl metacrilato amorfo grado extrusión/inyección, suministrado por la empresa Americana IDES. Este polímero tiene un IF de $16 \mathrm{~g} / 10 \mathrm{~min}\left(\mathrm{a} 230^{\circ} \mathrm{C} / 3.80 \mathrm{~kg}\right.$ ), una $\mathrm{Tg}$ de $100^{\circ} \mathrm{C}$ y una temperatura de fusión de $102^{\circ} \mathrm{C}$.

Procesamiento del polímero. El PMMA fue procesado cinco veces en una extrusora mono husillo 0 . Este modelo tiene un diámetro de husillo de $45 \mathrm{~mm}$, una relación $\mathrm{L} / \mathrm{D}$ de 30 y una restricción en la zona de dosificación ajustable entre 0 (máxima) y 56 (mínima). El sistema de calefacción de la extrusora tiene 8 zonas de calentamiento. Cada ciclo de procesamiento corresponde a la siguiente secuencia de operaciones: primer secado, extrusión, enfriamiento, reducción de tamaño o peletizado y segundo secado.

La extrusión del PMMA se llevó a cabo bajo las siguientes condiciones de operación: el perfil de temperatura fue 180/190/200/210/220/210/210/2 10 , con velocidades de procesamiento de $30 \mathrm{rpm}$, una restricción constante en la boquilla del extrusor de 10, y un tiempo de residencia de $2.7 \mathrm{~min}$. Para iniciar cada extrusión, el equipo trabaja sin material por un periodo de 10 min después de alcanzadas las condiciones de operación. Al cabo de este tiempo, el material se alimenta y se desechan los dos primeros kilos de material que salen de la extrusora para asegurar que no hay mezcla entre materiales procesados. Para la primera extrusión se alimenta $20 \mathrm{~kg}$ de polímero virgen. Para asegurar la reproducibilidad de los resultados, las condiciones de operación en cada uno de los cinco ciclos de procesamiento fueron las mismas, los tiempos de secado del material fueron iguales para todas las extrusiones $\left(1 \mathrm{~h} \mathrm{a} 80^{\circ} \mathrm{C}\right)$, y se utilizó el mismo peletizador sin modificar su velocidad.

Calorimetría diferencial de barrido, DSC. La determinación de la $\mathrm{Tg}$ del polímero virgen ( $\sin$ procesar) y extruido, se realizó en un calorímetro diferencial de barrido marca Netzsch DSC 200PC, de acuerdo a la norma ASTM D3418-08. Para el análisis se utilizó una masa de muestra entre 9.0 y $10.0 \mathrm{mg}$, que se sometió a un programa de calentamiento/enfriamiento, así: calentamiento de 20 a $160^{\circ} \mathrm{C}$, aquí la temperatura se mantuvo constante por $5 \mathrm{~min}$; después se enfrió de 160 a $20^{\circ} \mathrm{C}$. El ciclo de calentamiento/enfriamiento se realizó a $10^{\circ} \mathrm{C} / \mathrm{min}$ y en atmosfera de nitrógeno.

Índice de fluidez - IF. El análisis se realizó según la norma ASTM D 1238, en un plastómetro de extrusión marca Dynisco Polymer Test, modelo D4003. En este análisis se determinó la masa que fluye en un capilar en un tiempo de $10 \mathrm{~min}$, a $230^{\circ} \mathrm{C}$ con una masa del equipo de $3.80 \mathrm{~kg}$.

Poder calorífico - PC. Este parámetro se determinó de acuerdo a la norma ASTM D5865, empleando una Bomba Calorimétrica marca LECO AC 350, con un tamaño de muestra de $1 \mathrm{~g}$.

Análisis termogravimétrico - TGA. Este análisis se llevó a cabo en un Analizador Termogravimétrico SDT Q600 V20.9. Una masa de muestra entre 10 y $20 \mathrm{mg}$ se alimentó a un crisol de aluminio, la cual se calentó a 4 velocidades diferentes $(B=5$, $10,20 \mathrm{y} 40^{\circ} \mathrm{C} / \mathrm{min}$ ) desde temperatura ambiente hasta $700^{\circ} \mathrm{C}$ en atmosfera inerte, con un flujo de nitrógeno constante de $50 \mathrm{~mL} / \mathrm{min}$. A partir de los datos entregados por el equipo, se obtuvieron las gráficas de peso remanente, conversión y velocidad de pérdida de peso en función de la temperatura para cada velocidad de calentamiento a cada una de las muestra extruidas y a la muestra virgen.

Determinación del triplete cinético. Los valores de energía de activación $(E)$, factor de frecuencia $(A)$, y orden de reacción $(n)$ fueron calculados con los métodos descritos en la tabla 1. El $A$ es una medida de la probabilidad o fracción de colisiones entre las moléculas del polímero para que ocurra 
Tabla 1. Métodos cinéticos empleados para determinar el triplete cinético

\begin{tabular}{|c|c|c|c|c|}
\hline \multirow{2}{*}{ Método } & \multirow{2}{*}{ Ecuación } & \multirow{2}{*}{ Fuente } & \multicolumn{2}{|r|}{ Ejes regresión } \\
\hline & & & $x$ & $y$ \\
\hline $\begin{array}{l}\text { Flynn-Wall- } \\
\text { Ozawa-FWO }\end{array}$ & $\operatorname{Ln} B=\operatorname{Ln}\left(\frac{A E}{R F(a)}\right)-5.3305-\frac{1.052 E}{R T}$ & $\begin{array}{l}\text { Park et al., } \\
2000 a, b\end{array}$ & $\frac{1}{T}$ & $\ln B$ \\
\hline Friedman & $\ln \left(\frac{d a}{d t}\right)=\ln \left[A(1-\alpha)^{n}\right]-\frac{E}{R T}$ & $\begin{array}{l}\text { Kim y Oh, } \\
\quad 2005\end{array}$ & $\frac{1}{T}$ & $\ln \left(\frac{d \alpha}{d t}\right)$ \\
\hline $\begin{array}{l}\text { Gyulai- } \\
\text { Greenhow }\end{array}$ & $\ln B=\ln \left(\frac{A}{F(\alpha)}\right)+8.1614-0.915784 \ln E-\frac{621.302 E^{0.95823}}{T}$ & $\begin{array}{l}\text { Mallakpour } \\
\text { y Taghavi, } \\
2009\end{array}$ & $\frac{1}{T}$ & $\ln B$ \\
\hline $\begin{array}{l}\text { Kissinger- } \\
\text { Akahira-Sunose } \\
\quad-\text { KAS }\end{array}$ & $\ln \left(\frac{B}{T^{2}}\right)=\ln \left(\frac{A R}{E F(\alpha)}\right)-\frac{E}{R T}$ & $\begin{array}{l}\text { Aboulkas et } \\
\text { al., } 2010\end{array}$ & $\frac{1}{T}$ & $\ln \left(\frac{\mathrm{B}}{T^{2}}\right)$ \\
\hline Kissinger & $\ln \left(\frac{B}{T_{m}^{2}}\right)=\ln \frac{A R}{E}-\frac{E}{R} \frac{1}{t_{m}}$ & $\begin{array}{l}\text { Kim y Oh, } \\
\quad 2005\end{array}$ & $\frac{1}{T_{m}}$ & $\ln \left(\frac{\mathrm{B}}{T_{m}^{2}}\right)$ \\
\hline $\begin{array}{l}\text { Arrhenius } \\
\text { Diferencial }\end{array}$ & $\ln \left[\frac{1}{1-\alpha} \frac{d \alpha}{d T}\right]=\ln \frac{A}{B}-\frac{E}{R T}$ & $\begin{array}{l}\text { Wan et al., } \\
\quad 2005\end{array}$ & $\frac{1}{T}$ & $\ln \left[\frac{1}{1-\alpha} \frac{d \alpha}{d T}\right]$ \\
\hline $\begin{array}{l}\text { Arrhenius } \\
\text { Integral }\end{array}$ & $\ln [-\ln (1-\alpha)]=\ln \left[\frac{A R T^{2}}{B E}\left(1-\frac{2 R T}{E}\right)\right]-\frac{E}{R T}$ & $\begin{array}{l}\text { Wan et al., } \\
\quad 2005\end{array}$ & $\frac{1}{T}$ & $\ln [-\ln (1-\alpha)]$ \\
\hline Briodo & $\ln \left[-\ln \left(\frac{1}{1-\alpha}\right)\right]=\ln \left(\frac{A R T_{n}^{2}}{B E}\right)-\frac{E}{R T}$ & $\begin{array}{l}\text { Mano et al., } \\
2003\end{array}$ & $\frac{1}{T}$ & $\ln \left[\ln \left(\frac{1}{1-\alpha}\right)\right]$ \\
\hline Chang & $\ln \left[\frac{\frac{d \alpha}{d t}}{(1-\alpha)^{n}}\right]=\ln (A)-\frac{E}{R} *\left(\frac{1}{T}\right)$ & $\begin{array}{l}\text { Narayan et } \\
\text { al., } 2005\end{array}$ & $\frac{1}{T}$ & $\ln \left[\frac{\frac{d \alpha}{d t}}{(1-\alpha)^{n}}\right]$ \\
\hline Coats-Redfern & $\begin{array}{l}\ln \left[\frac{-\ln (1-\alpha)}{T^{2}}\right]=\ln \left[\frac{A R}{B E}\left(1-\frac{2 R T}{E}\right)\right]-\frac{E}{R T} \quad n=1 \\
\ln \left[\frac{1-(1-\alpha)^{1-n}}{T^{2}(1-n)}\right]=\ln \left[\frac{A R}{B E}\left(1-\frac{2 R T}{E}\right)\right]-\frac{E}{R T} \quad n \neq 1\end{array}$ & $\begin{array}{l}\text { Criado et } \\
\text { al., } 1987\end{array}$ & $\frac{1}{T}$ & $\begin{array}{l}\ln \left[\frac{-\ln (1-\alpha)}{r^{2}}\right] \quad n=1 \\
\ln \left[\frac{1-(1-\alpha)^{1-n}}{r^{2}(1-n)}\right] \quad n=1\end{array}$ \\
\hline Flynn-Wall & $\frac{E}{n R T_{m}^{2}\left(1-\alpha_{m}\right)^{n-1}}=\frac{A}{B} \exp \left(-\frac{E}{R T_{m}}\right)$ & $\begin{array}{l}\text { Wan et al., } \\
\quad 2005\end{array}$ & $\frac{1}{T_{m}}$ & $\frac{E}{n R T_{m}^{z}\left(1-\alpha_{m}\right)^{n-1}}$ \\
\hline $\begin{array}{l}\text { Horowitz- } \\
\text { Metzger }\end{array}$ & $\begin{array}{ll}\ln [-\ln (1-\alpha)]=\frac{E \theta}{R T_{m}^{2}} & n=1 \\
\ln \left[\frac{1-(1-\alpha)^{1-n}}{(1-n)}\right]=\ln \frac{A R T_{m}^{2}}{B E}-\frac{E}{R T_{m}}+\frac{E \theta}{R T_{m}^{2}} & n \neq 1\end{array}$ & $\begin{array}{l}\text { Kim y Oh, } \\
\quad 2005\end{array}$ & $\frac{1}{T_{m}^{2}}$ & $\begin{array}{l}\ln [-\ln (1-\alpha)] \quad n=1 \\
\ln \left[\frac{1-(1-\alpha)^{1-n}}{(1-n)}\right] n \neq 1\end{array}$ \\
\hline $\begin{array}{l}\text { MacCallum- } \\
\text { Tanner }\end{array}$ & $\ln F(\alpha)=\ln \left(\frac{A E}{R B}\right)-1,1124 E^{0,4351}-\left(\frac{449+217 E}{0.434}\right)\left(\frac{1}{T}\right)$ & $\begin{array}{l}\text { Mallakpour } \\
\text { y Taghavi, } \\
2009\end{array}$ & $\frac{1}{T_{m}^{2}}$ & $\ln F(\alpha)$ \\
\hline Madhusudanan & $\ln \left(\frac{F(\alpha)}{T^{1,921503}}\right)=\ln \left(\frac{A E}{B R}\right)+3,772049679-1,921503 \ln (E)-1,000953 \frac{E}{R}\left(\frac{1}{T}\right)$ & $\begin{array}{l}\text { Deng et al., } \\
2009\end{array}$ & $\frac{1}{T}$ & $\ln \left(\frac{F(\alpha)}{T^{1,921503}}\right)$ \\
\hline $\begin{array}{l}\text { Método } \\
\text { Dinámico }\end{array}$ & $\ln (B)=\ln (n)+(n-1) \ln \left(1-\alpha_{m}\right)+\ln (A)+\frac{3}{2} \ln \left(T_{m}\right)-\left(\frac{E}{R T_{m}}+\frac{1}{2}\right)-\frac{E}{R T_{m}}$ & $\begin{array}{l}\text { Kim y Oh, } \\
2005\end{array}$ & $\frac{1}{T_{m}}$ & $\ln (\mathrm{B})=\ln (n)$ \\
\hline
\end{tabular}

$T_{m}:$ Temperatura a la máxima velocidad de pérdida de peso, $\alpha_{m}:$ Conversión a $T_{m^{\prime}} \theta=T-T_{m}$, B: Velocidad de calentamiento, $F(\alpha)$ : Solución de la integral $\int_{0}^{\alpha} \frac{d \alpha}{(1-\alpha)^{n}}$

una reacción de degradación (Santos et al., 2006) y el $\mathrm{n}$ dependerá del método usado para determinar la cinética de degradación térmica (isoconversionales y no isoconversionales). Para el ajuste de datos de temperatura $(T)$, conversión $(\alpha)$ y velocidad de calentamiento $(B)$, se utilizó el software de licencia libre Scilab-5.4.0 en versión para Windows de 64 bits. En este software se creó una función para realizar un ajuste lineal entre un conjunto de pares ordenados, los cuales son función del método 
utilizado (ver tabla 1). Así mismo se creó un programa para los métodos no isoconversionales e isoconversionales, validado mediante Excel.

\section{Resultados y discusión}

Caracterización. Se determinó la $T g, I F$ y $P C$ superior, para el polímero sin procesar y procesado de 1 a 5 extrusiones. Se encontró valores de $T g$ de $98,102,102,102$ y $100^{\circ} \mathrm{C}$, de IF de 18.50 , $18.40,17.70,16.80,16.80$ y $16.50 \mathrm{~g} / 10 \mathrm{~min}, \mathrm{y}$ de PC de 26135, 26101, 26087, 26075 y $26056 \mathrm{~kJ} /$ $\mathrm{kg}$, para las muestra extruidas entre 1 y 5 ciclos, respectivamente. Para la muestra sin procesar éstos valores fueron: $100^{\circ} \mathrm{C}, 20.50 \mathrm{~g} / 10 \mathrm{~min}$ y $26147 \mathrm{~kJ} /$ $\mathrm{kg}$. Se observa que la $T g$ no varía considerablemente con el número de extrusiones, los datos sugieren que el reprocesamiento no afecta dicho parámetro. Respecto al $P C$ se observa que disminuye con el número de reprocesamientos. Este comportamiento era de esperarse, dado que el polímero experimenta termodegradación de la estructura molecular. Sin embargo, la reducción de este parámetro es menor al $0.50 \%$, por lo que estadísticamente indica que no es representativo el efecto del reprocesamiento sobre el $P C$.

Análisis termogravimétrico. En la figura 1 se presentan las curvas a) $T G$ y b) $D T G$ para la extrusión 5 a diferentes $B$. En la figura la se observa que los termogramas se desplazan hacia la derecha con la $B$ (tendencia similar se aprecia en las curvas de conversión). Este mismo comportamiento se observó en las curvas $T G$ para las otras extrusiones. Estos resultados concuerdan a los reportado en la literatura (Kim et al., 2000). Esta tendencia posiblemente se debe a que al aumentar la $B$ : i) el tiempo de reacción se reduce, dado que la temperatura final de análisis se alcanza rápidamente, $\mathrm{y}$ ii) posiblemente se induce a un cambio en el mecanismo de reacción (Park et al., 2000b).

En la figura 1b se observa que la curva DTG se desplaza alejándose del eje de las ordenadas,
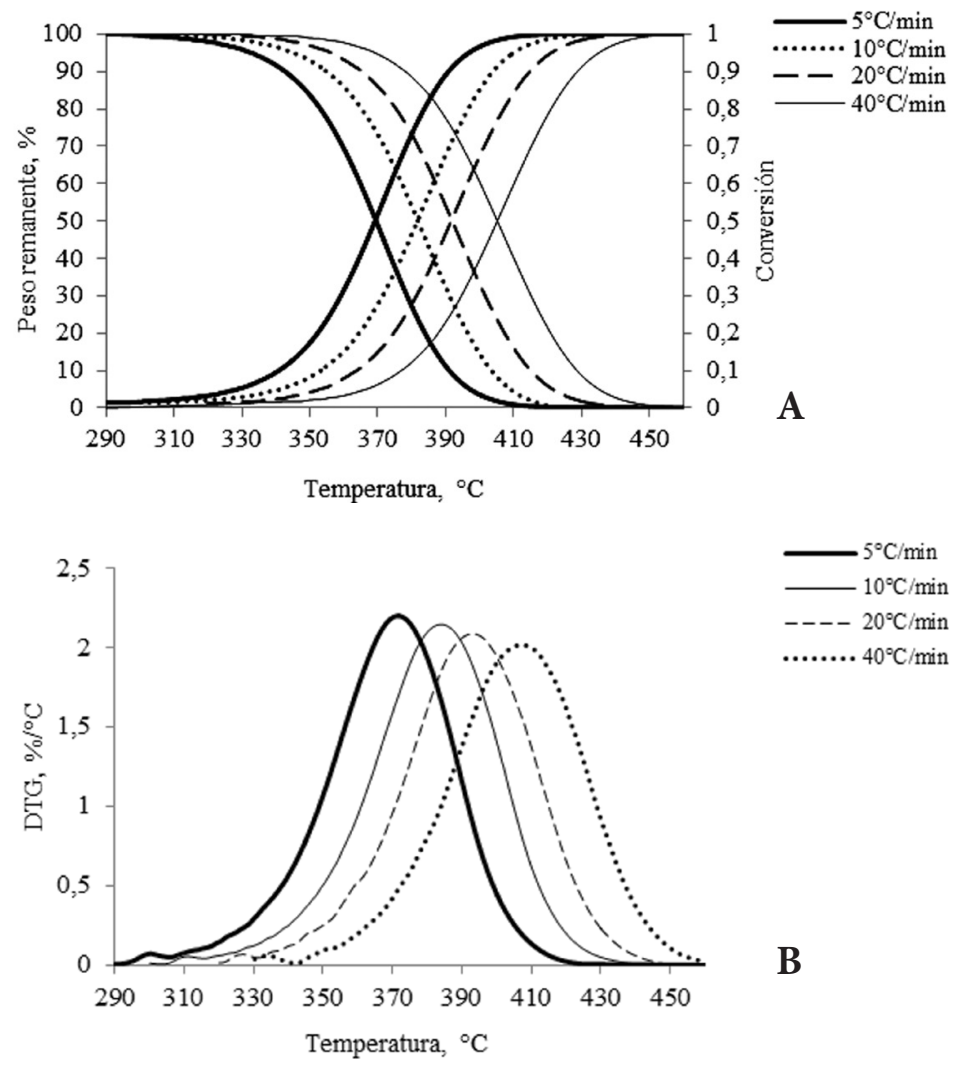

Figura 1. Curvas a) TG y b) DTG para la extrusión 5 variando la velocidad de calentamiento 
aumentando la temperatura máxima de pérdida de peso y disminuyendo la velocidad de degradación dada por la altura del pico, con el incremento en la $B$. Esto es causado por la reducción del tiempo de reacción (Kim et al., 2000).

En la figura 2 se presentan las curvas a) TG y b) DTG a $10^{\circ} \mathrm{C} / \mathrm{min}$ para la muestra sin procesar y para los 5 reprocesamientos. En la figura 2a Se observa que el perfil de degradación térmica, al igual que la curva de conversión, del PMMA presenta una leve tendencia hacia la derecha, la cual no es apreciable porque los perfiles son similares, esto sugiere que la degradación térmica de un reprocesamiento a otro para los cinco ciclos no afecta considerablemente las propiedades térmicas del polímero. Esto también indica que el mecanismo de degradación térmica del PMMA es similar cuando experimenta cinco ciclos sucesivos de extrusión a condiciones de procesamiento real en la actual industria. En la figura $2 \mathrm{~b}$ se muestra que no hay diferencias apreciables en los termogramas de las muestra al experimentar cambios térmicos debido al reprocesamiento, lo cual indica que el mecanismo de degradación térmica del PMMA virgen y reprocesado es similar (Denq et al., 1997). Este mismo comportamiento se observó para las diferentes B. También se observa que las curvas DTG presentan un único pico, que sugiere que la degradación térmica de las muestras reprocesadas se degradan en un proceso de una sola etapa (Aboulkas et al., 2010). Comportamiento similar se obtiene a diferentes B (figura 1b).

La figura 3 muestra la variación de Tm con el número de procesamientos, a cada $\mathrm{B}$. Se observa que Tm para una extrusión determinada, aumenta con la B, pero permanece casi constante con el número de procesamientos al fijar la $\mathrm{B}$. Esto indica que, si se considera la Tm como un parámetro para determinar la termodegradación, el PMMA sufre una degradación despreciable con el reprocesamiento, confirmando de esta manera los resultados de $\mathrm{PC}$.
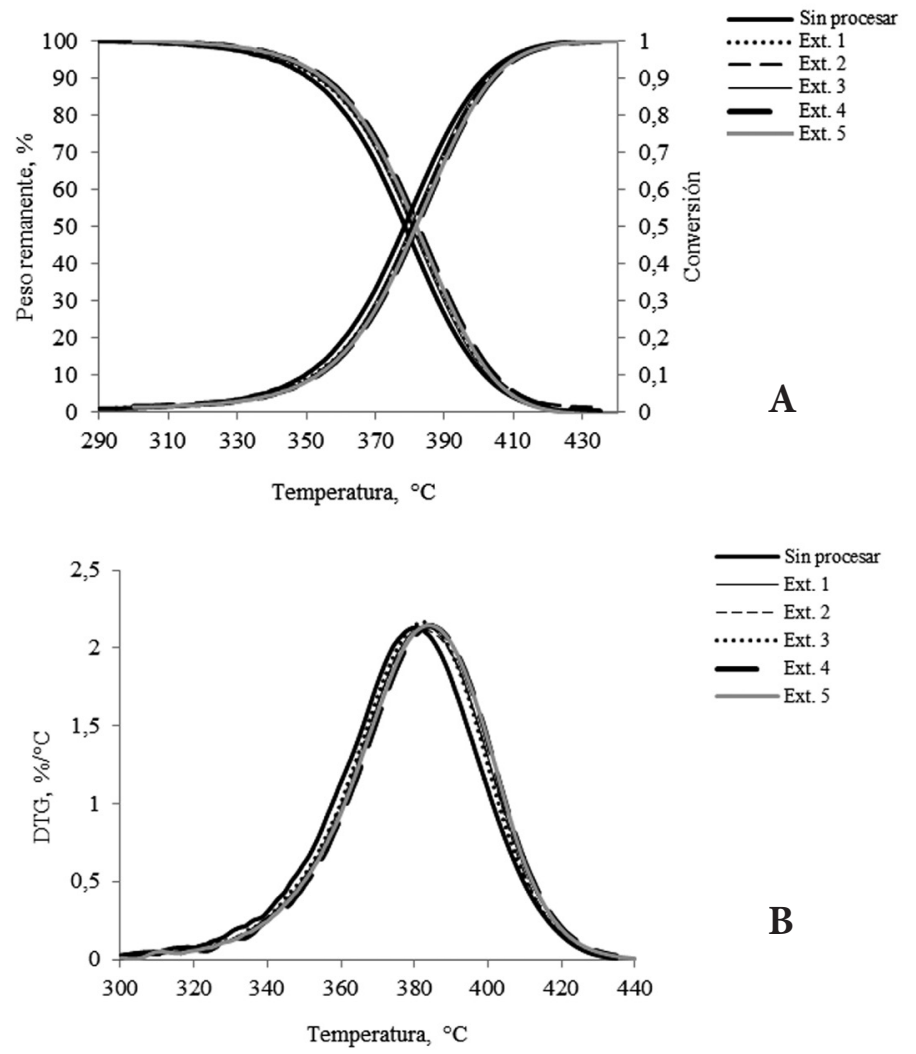

Figura 2. Curvas a) $T G$ y b) DTG a $10^{\circ} \mathrm{C} / \mathrm{min}$ variando el número de extrusiones 


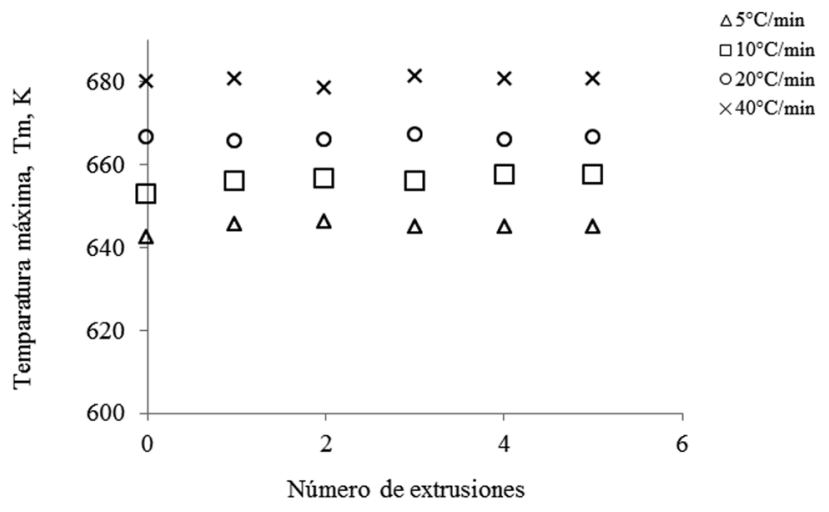

Figura 3. Variación de la temperatura máxima de pérdida de peso con el número de extrusiones a diferentes velocidades de calentamiento

Tabla 2. Resultados triplete cinético para un orden de reacción de la unidad métodos no isoconversionales

\begin{tabular}{|c|c|c|c|c|c|c|c|}
\hline \multirow{2}{*}{$\begin{array}{c}\text { Muestra, } \\
B\left({ }^{\circ} \mathrm{C} / \mathrm{min}\right)\end{array}$} & \multicolumn{2}{|c|}{ Briodo } & \multicolumn{2}{|c|}{ Diferencial Arrhenius } & \multicolumn{2}{|c|}{ Integral de Arrhenius } & \multirow{2}{*}{$\begin{array}{c}\text { Horowitz } \\
\text { Metzger } \\
E(\mathrm{~kJ} / \mathrm{mol})\end{array}$} \\
\hline & $E(k J / m o l)$ & $\operatorname{Ln} A\left(\min ^{-1}\right)$ & $E(\mathrm{~kJ} / \mathrm{mol})$ & $\operatorname{Ln} A\left(\min ^{-1}\right)$ & $E(k J / m o l)$ & $\operatorname{Ln} A\left(\min ^{-1}\right)$ & \\
\hline Sin Proce., 5 & 161.25 & 29.62 & 182.38 & 32.53 & 161.25 & 29.41 & 60.42 \\
\hline 10 & 174.10 & 32.08 & 191.86 & 34.28 & 174.10 & 31.85 & 67.36 \\
\hline 20 & 174.81 & 32.19 & 206.94 & 36.97 & 174.81 & 31.97 & 67.53 \\
\hline 40 & 189.69 & 34.95 & 214.66 & 38.32 & 189.69 & 34.75 & 75.95 \\
\hline Ext. 1, 5 & 179.10 & 32.08 & 193.15 & 34.26 & 179.10 & 32.54 & 67.30 \\
\hline 10 & 174.75 & 32.08 & 189.27 & 33.71 & 174.75 & 31.87 & 67.71 \\
\hline 20 & 179.37 & 33.10 & 202.01 & 36.28 & 179.37 & 32.89 & 71.64 \\
\hline 40 & 185.85 & 34.15 & 190.06 & 33.88 & 185.85 & 33.93 & 75.51 \\
\hline Ext. 2, 5 & 165.42 & 30.23 & 197.67 & 35.13 & 165.42 & 30.01 & 62.20 \\
\hline 10 & 183.79 & 33.78 & 206.78 & 36.90 & 183.79 & 33.57 & 70.53 \\
\hline 20 & 204.88 & 37.65 & 207.04 & 36.91 & 204.88 & 37.42 & 79.30 \\
\hline 40 & 196.82 & 36.20 & 209.11 & 37.34 & 196.82 & 35.99 & 79.89 \\
\hline Ext. 3, 5 & 180.34 & 33.09 & 189.19 & 33.65 & 180.34 & 32.89 & 64.45 \\
\hline 10 & 187.17 & 34.45 & 202.85 & 36.26 & 187.17 & 34.25 & 72.09 \\
\hline 20 & 179.72 & 33.21 & 195.93 & 35.09 & 179.72 & 33.01 & 70.93 \\
\hline 40 & 191.09 & 35.15 & 208.69 & 37.22 & 191.09 & 34.95 & 76.28 \\
\hline Ext. 4,5 & 170.31 & 31.20 & 183.56 & 32.60 & 170.31 & 31.00 & 63.62 \\
\hline 10 & 176.04 & 32.27 & 201.70 & 35.89 & 176.04 & 32.06 & 68.00 \\
\hline 20 & 193.45 & 35.61 & 193.84 & 34.60 & 193.45 & 35.37 & 75.58 \\
\hline 40 & 189.08 & 34.78 & 200.52 & 35.79 & 189.08 & 34.58 & 76.78 \\
\hline Ext. 5, 5 & 176.38 & 32.34 & 197.20 & 35.09 & 176.38 & 32.12 & 65.63 \\
\hline 10 & 178.70 & 32.76 & 205.58 & 36.54 & 178.70 & 32.53 & 68.43 \\
\hline 20 & 200.60 & 36.92 & 206.62 & 36.87 & 200.60 & 36.69 & 77.14 \\
\hline 40 & 204.93 & 37.62 & 216.01 & 38.50 & 204.93 & 37.42 & 82.16 \\
\hline
\end{tabular}


Determinación del Triplete Cinético. En la tabla 1 se muestra las variables de cada ecuación para realizar la regresión. En la tabla 2 se presenta los resultados del triplete cinético, a las cuatro B $\left(5,10,20\right.$ y $\left.40^{\circ} \mathrm{C} / \mathrm{min}\right)$, para los métodos no isoconversionales que asumen reacción de primer orden. En esta se observa que tanto la E como el A tienden a aumentar con el incremento en la B. También se aprecia que en la mayoría de los reprocesamientos la probabilidad de colisión aumenta con la B, de tal manera que aumenta la energía interna y la energía cinética de las moléculas en un menor tiempo, por lo tanto, se tiene un mayor número de moléculas con la energía suficiente para superar el estado de transición y así reaccionar. Es de destacar que el método Horowitz-Metzger reporta los valores más bajos para cualquier $\mathrm{B}$ y reprocesamiento (Tabla 2). En los métodos no isoconversionales, con $n$ diferente a la unidad, se encontró que $n$ se ve ligeramente afectado por la B (Park et al., 2000b), presentándose aumenta hasta un máximo a una $\mathrm{B}$ de $20^{\circ} \mathrm{C} / \mathrm{min}$ y cae a $40^{\circ} \mathrm{C} / \mathrm{min}$.
También se encontró que al fijar B y la etapa de reprocesamiento, el $\mathrm{n}$ es similar en los métodos MacCallum-Tanner, Madhusudanan y Coats Redfern. Este mismo comportamiento se presentó para A. En el método dinámico el A no varía con la B. Los resultados del A para los métodos isoconversionales Kissinger, KAS, FWO, Gyulai y Friedman se muestran en la tabla 3. Los valores de $\mathrm{n}$ solo se muestran para el método Friedman, los otros métodos consideran $\mathrm{n}$ igual a la unidad. El n para este método varía ligeramente con el reprocesamiento, con valores cercanos a la unidad (1.06 - 1.10), lo cual indica una cinética de cuasiprimer orden.

En la figura 4 se presenta la variación de la $E$ con $\alpha$ para las muestras reprocesadas empleando los métodos isoconversionales a) KAS y b) FWO. Aquí se aprecia que la $E$ aumenta con la $\alpha$, excepto para el primer reprocesamiento que permanece casi constante, esto indica que la degradación térmica del polímero se retrasa o retarda debido a que el material se descompone volviéndose más
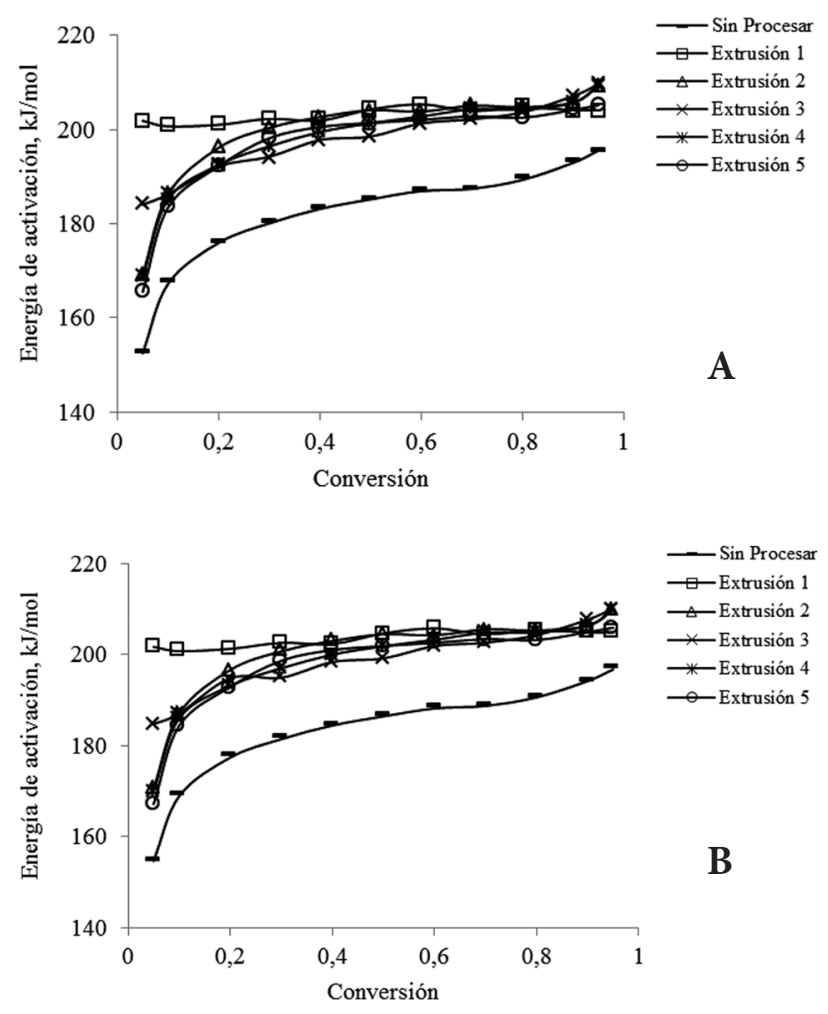

Figura 4. Cambio de la energía de activación con la conversión para cada ciclo de extrusión métodos a) KAS y b) FWO 
Tabla 3. Resultados triplete cinético para un orden de reacción de la unidad métodos isoconversionales

\begin{tabular}{|c|c|c|c|c|c|c|}
\hline \multirow{2}{*}{ Muestra } & \multirow{2}{*}{$\begin{array}{c}\text { Kissinger } \\
\operatorname{Ln} A\left(\text { min }^{-1}\right)\end{array}$} & \multirow{2}{*}{$\begin{array}{c}K A S \\
\operatorname{Ln} A\left(\min ^{-1}\right)\end{array}$} & \multirow{2}{*}{$\begin{array}{c}F W O \\
\operatorname{Ln} A\left(\min ^{-1}\right) \\
\end{array}$} & \multirow{2}{*}{$\begin{array}{c}\text { Gyulai } \\
\operatorname{Ln} A\left(\text { min }^{-1}\right)\end{array}$} & \multicolumn{2}{|c|}{ Friedman } \\
\hline & & & & & $\operatorname{Ln} A(\min -1)$ & $n$ \\
\hline Sin Proces. & 33.70 & 32.35 & 32.66 & 32.62 & 34.28 & 1.00 \\
\hline Ext. 1 & 37.84 & 36.37 & 36.47 & 36.65 & 35.72 & 1.06 \\
\hline Ext. 2 & 40.09 & 35.51 & 35.66 & 35.79 & 36.61 & 1.06 \\
\hline Ext. 3 & 35.95 & 35.39 & 35.54 & 35.66 & 37.16 & 1.08 \\
\hline Ext. 4 & 37.48 & 35.22 & 35.38 & 35.49 & 35.80 & 1.10 \\
\hline Ext. 5 & 37.24 & 35.01 & 35.18 & 35.29 & 35.09 & 1.05 \\
\hline
\end{tabular}

refractario cuando la $T$ aumenta (Badia et al., 2012) y como consecuencia se aumenta su estabilidad térmica (Santos et al., 2006). Esto también se observa al comparar $E$ de las muestras reprocesadas con el polímero virgen, de igual manera $E$ aumenta en el rango de 165 y $195 \mathrm{~kJ} / \mathrm{mol}$ para $\alpha$ entre 0.05 y 0.30 , y permanece casi constante entre 0.35 y 0.95 . A pesar de este incremento, la variación de $\mathrm{E}$ no se considera tan relevante, por lo cual se sugiere que el proceso de descomposición del polímero ocurre en una sola etapa de reacción (Aboulkas et al., 2010).
La variación de la E con el número de extrusiones se muestra en la figura 5 para los métodos a) isoconversionales y $\mathrm{b}$ ) no isoconversionales a $10^{\circ} \mathrm{C} / \mathrm{min}$. La tendencia es la misma para las demás $\mathrm{B}$. Al comparar el comportamiento de los métodos isoconversionales se observa que los métodos KAS, FWO y Gyulai tienen la misma tendencia, mientras que el método Kissinger presenta un comportamiento aproximadamente sinusoidal con máximos en los reprocesamientos 2 y 4. En los métodos KAS, FWO y Gyulai
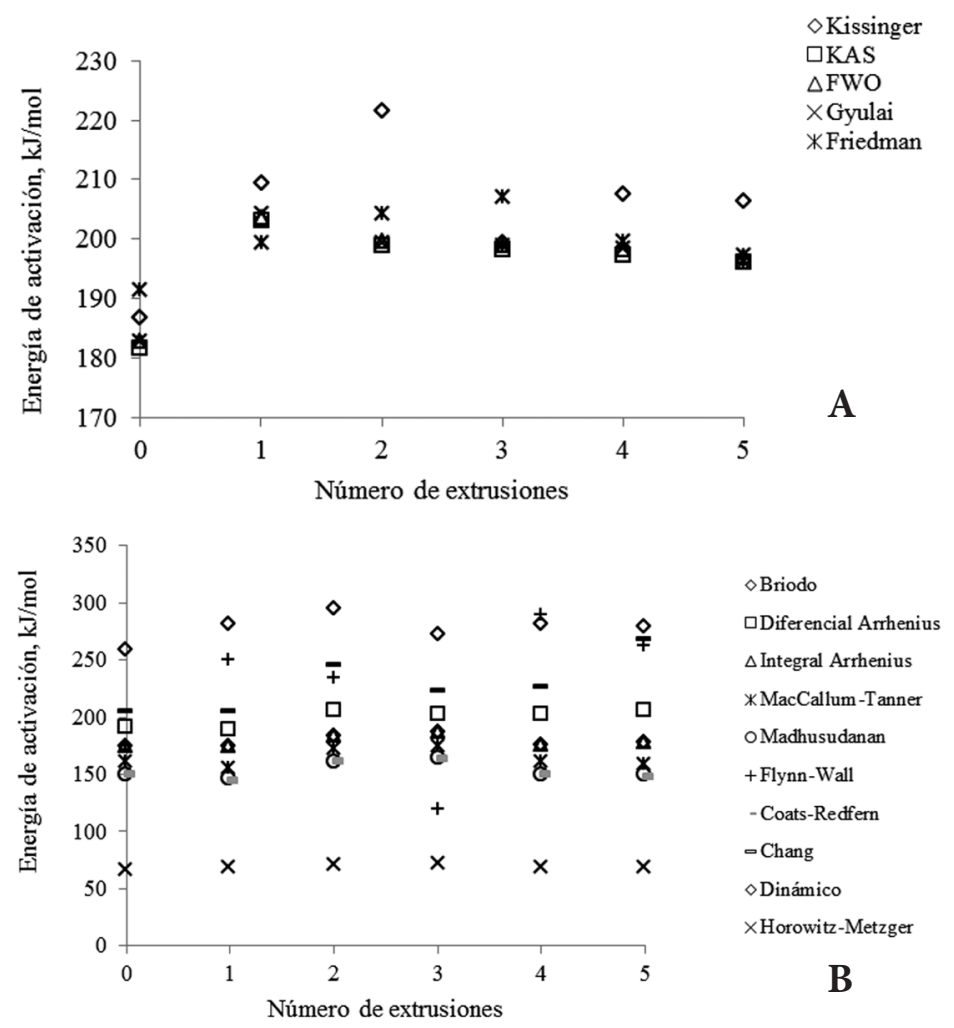

Figura 5. Variación de E con el número de extrusiones para los métodos a) isoconversionales y b) no isoconversionales a $10^{\circ} \mathrm{C} / \mathrm{min}$ 
E aumenta de 182 a $205 \mathrm{~kJ} / \mathrm{mol}$ en el primer reprocesamiento, que luego disminuye a un valor de $195 \mathrm{~kJ} / \mathrm{mol}$ en el reprocesamiento 5. Esto es consistente con la descomposición térmica y por cizalla del material, que toma lugar durante el reprocesamiento (Pérez et al., 2010). En la figura 5.b se aprecia que los métodos de Briodo e integral de Arrhenius y los métodos Madhusudanan y Coats-Redfern, producen resultados exactamente iguales. También se observa la misma tendencia de la mayoría de los métodos, y se presenta la siguiente variación de E: Dinámico $>$ Chang $>$ Diferencial Arrhenius $>$ Briodo $=$ Integral Arrhenius $>$ MacCallum-Tanner $>$ Madhusudanan $=$ Coats-Redfern $>$ HorowitzMetzger. De igual manera se ve que los métodos diferenciales (Diferencial Arrhenius, FlynnWall, Chang y Dinámico) arrojaron valores de E mayores a los métodos integrales (Briodo, Integral de Arrhenius, Horowitz-Metzger, MacCallumTanner, Madhusudanan y Coats-Redfern). El método Flynn-Wall presenta un comportamiento sinusoidal diferente a los demás métodos.

En cuanto al n, la figura 6 muestra su variación con el reprocesamiento, a una $\mathrm{B}$ de $10^{\circ} \mathrm{C} / \mathrm{min}$, para los métodos no isoconversionales que asumen $\mathrm{n}$ diferente de la unidad. Los métodos Diferencial e Integral de Arrhenius, Briodo, y HorowitzMetzger, asumen cinética de primer orden. El n en los métodos Madhusudanan, Coats-Redfern y MacCallum-Tanner tiene la misma variación con valores entre 0.70 y 0.90 , mientras que el método Dinámico reporta $\mathrm{n}$ entre 1.45 y 1.55 . El método
Chang muestra una tendencia a aumentar el $\mathrm{n}$ entre 1.10 y 1.50 .

El triplete cinético obtenido por todos los métodos fue satisfactorio dado que presentaron coeficientes de correlación superiores a 0.90 . Sin embargo, por análisis estadístico de minimización de residuos los métodos isoconversionales son los más adecuados para encontrar los parámetros del triplete cinético, y entre estos el método de Kissinger es el mejor.

\section{Conclusiones}

Se estableció que la probabilidad o fracción de colisión (factor pre-exponencial o factor de frecuencia) aumenta con la velocidad de calentamiento, debido a que al aumentar la velocidad de calentamiento aumenta la energía interna y la energía cinética de las moléculas en un menor tiempo.

El reprocesamiento efectivamente afecta la degradación térmica del PMMA, sin embargo, para 5 extrusiones su degradación no es muy notable dado que su estabilidad térmica provoca que el polímero obtenido tenga características refractarias. Esto se confirma con el leve incremento de la energía de activación al someterse entre uno y cinco reprocesamientos.

Los métodos isoconversionales son los más adecuados para encontrar los valores del triplete cinético en la degradación térmica por

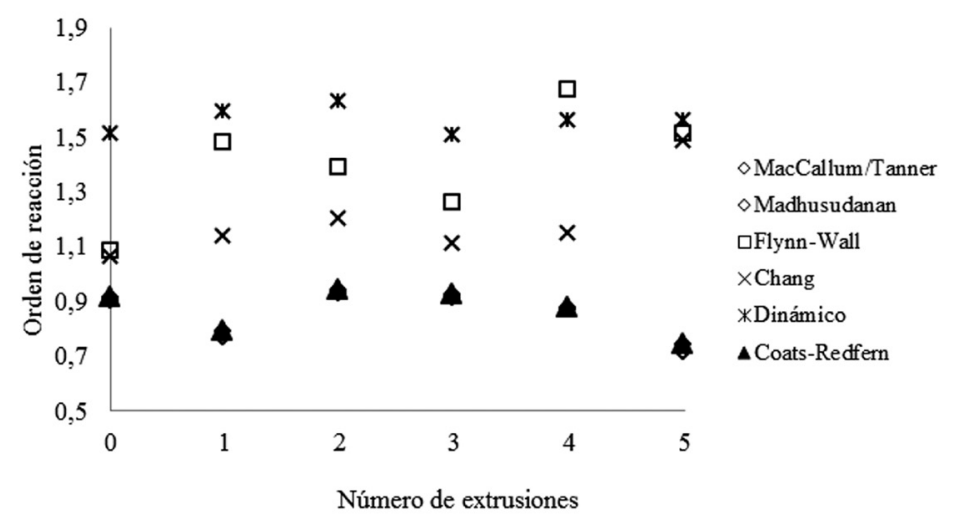

Figura 6. Orden de reacción a diferentes extrusiones para los métodos no isoconversionales (con $n \neq 1$ ) a $10^{\circ} \mathrm{C} / \mathrm{min}$ 
reprocesamiento del PMMA; y entre ellos el método de Kissinger es el mejor método para obtener dichos parámetros.

\section{Agradecimientos}

Los autores agradecen a la Dirección de Investigación de Manizales DIMA, de la Universidad Nacional de Colombia sede Manizales, por el apoyo al semillero de investigación "Estudio cinético de la degradación térmica de PMMA" con código 15704. Igualmente se agradece al profesor Tim Osswald del Polymer Engineering Center, de la Universidad de Wisconsin, Madison, USA.

\section{Referencias bibliográficas}

Abad, M.J., Ares, A., Barral, L., Cano, J., Díez, F.J., García-Garabal, S., López, J., \& Ramírez, C., (2004). Effect of a moisture of stabilizer on the structure and mechanical properties of polyethylene during reprocessing. Journal of Applied Polymer Science 92, 3910-3916.

Aboulkas, A., El Harfi, K. \& El Bouadili, A. (2010). Thermal degradation behaviors of polyethylene and PS. Part I: Pyrolysis. Energy Conversion and Management 51, 1363-1369.

Badia, J.D., Santonja-Blasco, L., Martínez-Felipe,A., \& Ribes-Greus A. (2012). Reprocessed polyactide: studies of thermo-oxidative decomposition. Bioresource Technology 114, 622-628.

Brandrup, J., Bittner M., Menges, G., \& Michaeli, W. (1996) Recycling and Recovery of Plastics. Hanser Publisers. 297.

Carrasco, F., Pages, P., Gómez-Pérez, J., Santana, O., \& Maspoch, ML. (2010). Processing of poly(lactic acid): characterization of chemical structure, thermal stability and mechanical properties. Polymer Degradation and Stability 95, 116-125.

Criado, J., Ortega, A., Roquerol, J., \& Roquerol, F. (1987). Un nuevo método de análisis térmico: el análisis térmico a velocidad de transformación controlada (ATVC). II. Boletín de la Sociedad Española de Cerámica y Vidrio 26, 3-11.
Dahiya, J.B., Kumar, K., Muller-Hagedorn M., \& Bockhorn H., (2008). Kinetics of isothermal and non-isothermal degradation of cellulose: modelbased and model-free methods. Society of chemical Industry, Polymer International 57, 722-729.

Deng, C., Cai, J., \& Liu, R., (2009). Kinetic analysis of solid-state reactions: evaluation of approximations to temperature integral and their applications. Solid State Science 11, 1375-1379.

Denq B.L., Chiu W.Y., \& Lin K.F., (1997). Kinetic model of thermal degradation of polymers for Non-isothermal process. Journal of Applied Polymer Science 66, 1855-1868.

Grellmann, W., \& Seidler, S., (2007). Polymer Testing. Munich: Editorial Hanser.

Holland,B.J. \& Hay, J.N., (2001). The kinetics and mechanisms of the thermal degradation of poly(methyl methacrylate) studied by thermal analysis-Fourier transform infrared spectroscopy. Polymer 42, 48254835.

Jitendra, K., Pondey, K., Ragunatha, A., Pratheep, K., \& Singh, R.P., (2005). An overview on the degradability of polymer nano-composites. Polymer Degradation and Stability 85, 234-250.

Kim, H. T., \& Oh, S., (2005). Kinetics of thermal degradation of waste polypropylene and highdensity polyethylene. Journal of Industrial and Engineering Chemistry 11 (5), 648-656.

Kim, W., Kim, S. D., \& Lee, S. B., (2000). Kinetic characterization of thermal degradation process for commercial rubbers. Journal of Industrial and Engineering Chemistry 6 (5), 348-355.

Mallakpour, S., \& Taghavi, M., (2009). The accuracy of approximation equations in the study of thermal decomposition behavior of some systhesized optically active polyamides. Iranian Polymer Journal 18 (11), 857-872.

Mano, J., Koniarova, D., \& Reis, R. L., (2003). Thermal properties of thermoplastic starch/ 
synthetic polymer blends with potential biomedical applicability. Journal of Materials Science: Materials in Medicine 14, 127-135.

Martins, M., \& De Paoli, M.A., (2002). Polypropilene compounding with post-consumer material: II. Reprocessing. Polymer Degradation and Stability 78, 491-495.

Mendes, A.A., Cunha, A.M., \& Bernardo, C.A., (2011). Study of the degradation mechanisms of polyethylene during reprocessing. Polymer Degradation and Stability 96, 1125-1133.

Mothé, C.G., De Araujo, C.R., De Oliverira, M.A., \& Yoshida, M.I., (2002). Thermal decomposition kinetics of polyurethane-composites with bagasse of sugar cane. Journal of Thermal Analysis and Calorimetry 67, 305-312.

Narayan, R., Chattopadhyay, D. K., Sreedhar, B., Raju, K. V. S. N., Mallikarjuna, N. N., \& Aminabhavi, T. M., (2005). Degradation profiles of polyester-urethane and polyester-melamine (HP-HMMM) coatings: a thermal study. Journal of Applied Polymer Science 97, 518-526.

Park, J. W., Oh, S.C., Lee, H.P., Kim, H.T., \& Yoo, K.O., (2000b). A kinetic analysis of thermal decomposition of polymer using a dynamic model. Polymer Degradation and Stability 67, 535-540.

Park, J.W., Oh, S.C., Lee, H.P., Kim, H.T., \& Yoo, K.O., (2000a). Kinetic analysis of thermal decomposition of polymer using a dynamic model. Korean Journal of Chem. Eng. 17, 489-496.

Pérez, J.M., Vilas, J.L., Laza, J.M., Arnáiz, S., Milangos, F., Bilbao, E., Rodríguez, M., \& León, L.M., (2010). Effect of reprocessing and accelerated ageing on thermal and mechanical polycarbonate properties. Journal of Materials Processing Technology 210, 727-733.

Santos, J.C., Dos Santos, I.M., DeSousa, A.G., Prasad, S., \& Dos Santos, A.V., (2006). Thermal stability and kinetic study on thermal decomposition of commercial edible oils by thermogravimetry. Journal of Food Science 67 (4), 1393-1398.

Wan, W. B., Ani, F. N., \& Masjuki, H. H., (2005). Thermal stability evaluation of palm oil as energy transport media. Energy Conversion \& Management 46, 2198-2215.

Wilichowski, P.A., (2004). Comparison of the effect of smooth and grooved feed extrusion on HDPE recyclability. Tesis de maestría, Departamento de Ingeniería Mecánica, Universidad de Wisconsin, Madison, USA. 